\title{
Using Strings for On-line Handwriting Shape Matching: A New Weighted Edit Distance
}

\author{
Claudio De Stefano ${ }^{1}$, Marco Garruto ${ }^{2}$, Luis Lapresa ${ }^{3}$, and Angelo Marcelli ${ }^{2}$ \\ ${ }^{1}$ Dipartimento di Automazione, \\ Elettromagnetismo Ingegneria dell'Informazione e \\ Matematica Industriale, Università di Cassino, \\ Via Di Biasio, 4303043 Cassino (FR), Italy \\ destefano@unicas.it \\ ${ }^{2}$ Dipartimento di Ingegneria dell'Informazione ed Ingegneria Elettrica, \\ Università di Salerno, Via Ponte Don Melillo, 84084 Fisciano (SA), Italy \\ marcounisa@libero.it, amarcelli@unisa.it \\ 3 Departament de Ciències Matemàtiques i Informàtica, \\ Universitat de les Illes Balears, Carretera de Valldemossa, \\ Km 7,5 07122 Palma (Illes Balears), Spain \\ lapresa@gmail.com
}

\begin{abstract}
Edit Distance has been widely studied and successfully applied in a large variety of application domains and many techniques based on this concept have been proposed in the literature. These techniques share the property that, in case of patterns having different lengths, a number of symbols are introduced in the shortest one, or deleted from the longest one, until both patterns have the same length. In case of applications in which strings are used for shape description, however, this property may introduce distortions in the shape, resulting in a distance measure not reflecting the perceived similarity between the shapes to compare. Moving from this consideration, we propose a new edit distance, called Weighted Edit Distance that does not require the introduction or the deletion of any symbol. Preliminary experiments performed by comparing our technique with the Normalized Edit Distance and the Markov Edit Distance have shown very encouraging results.
\end{abstract}

\section{Introduction}

Edit Distance has been widely studied and successfully applied in a large variety of application domains. In fact, in the applications in which matching, detection or recognition of patterns are of primary interest, a key role is played by the way in which the similarity or the dissimilarity between patterns is measured: in this context, edit distance techniques offer an effective and computationally efficient way of performing such a measure, and it has been demonstrated that their applicability is not limited to alphabet-based strings in text processing, but they can be profitably used in a multitude of different applications. Examples include genome representation in bioinformatics [1], message codes in information theory [2] and sound information in speech processing [3]. Moreover, the concept of edit distance has been widely used in many research disciplines of pattern recognition, image processing and computer vision $[4,5]$. 
The Edit distance between two strings was originally introduced in 1966 by Levenstein [6] and was defined as the minimum number of changes required to transform one string into another, where each change may be the insertion of a symbol, the deletion of a symbol or the substitution of a symbol with another. The greater the number of changes, the more different the strings are. In its original definition, called Levenstein Distance (LD), the changes are assumed to have the same (unitary) cost.

An obvious drawback of this definition is related to the fact that in real application the cost associated to a change may be different depending on the symbol inserted, deleted or substituted. To overcome this problem, a more general cost function has been introduced that allow to assign a different cost to each substitution of a symbol into another [7, 8]. The edit distance obtained in this way is called Generalized Levenstein Distance (GLD).

Another relevant problem encountered while using both LD and GLD is that such measures do not take into account the length of the patterns. This aspect may leads to meaningless measures in case of patterns having significantly different lengths: it is generally agreed that, for instance, two strings of length 4 having an edit distance equal to 2 should be considered much more dissimilar than two strings of length 100 having the same edit distance equal to 2. To solve this problem, Marzal and Vidal developed a Normalized Edit Distance (NED) in which the distance between two pattern is normalized by the length of the edit path [9]. In their work, they have also shown that NED outperforms any other normalization technique obtained by first computing the non normalized edit distance and then dividing by the length of the path.

Finally, to exploit the statistical dependencies among the values assumed by adjacent positions in the strings, an edit distance based on Markov Random Field, and therefore called Markov Edit Distance (MED), has been recently proposed [10].

Summarizing, the various edit distance techniques proposed in the literature differ for the way in which symbols to delete are selected, for the way in which both symbols to introduce and their position are chosen, and for the costs associated to each elementary operation (insertion, deletion and substitution).

Such techniques, however, share the property that, in case of patterns having different lengths, a number of symbols are introduced in the shortest one, or deleted from the longest one, until both patterns have the same length. This property, may have undesirable effects in case of application in which strings are used for shape description: in such cases, in fact, introducing or deleting a symbol may distort the corresponding shape resulting in a distance measure not reflecting the perceived distance between the shapes to compare.

Moving from these considerations, we propose a new edit distance, called Weighted Edit Distance (WED), based on the concept of string stretching: we do not introduce or delete any symbol in the strings to compare but simply extend or stretch the shortest string in such a way that each symbol of this string is compared with one or more symbols of the other, depending on the ratio $r$ between the lengths of the two strings. The edit distance is then computed by summing the cost of substitution of each compared pair of symbols, weighted by a coefficient whose value depends on both the position of the symbols in the two strings, and the value of $r$. A normalization is also applied to overcome the problems previously discussed. 
We have used WED in the framework of an on-line handwriting recognition system. In particular, we have developed an on-line handwriting segmentation method $[11,12]$ which allow to extract the elementary strokes a word is composed of, and provides segmentation points that are very accurate and stable, i.e. very invariant with respect to non significant variations in the shape of the ink. Each elementary stroke has been described by computing the arclength [13] representation and quantizing the values of the angles into 16 intervals encoded by one of the letter in the subset [A-P]. By this encoding, the shape of each elementary stroke is described by a string of characters.

Preliminary experiments performed by comparing our technique with the Normalized Edit Distance and the Markov Edit Distance have shown very encouraging results.

The remaining of the paper is organized as follows: Section 2 illustrates our method for on-line handwriting shape description. Section 3 describes the proposed Weighted Edit Distance technique. Preliminary experimental results and some concluding remarks are eventually left to Section 4.

\section{The Shape Description Method}

Studies on visual perception have shown that curvature plays a key role in our perception of shape and its organization into parts [14]. Therefore, since the sixties many efforts have been made to develop algorithms for computing the curvature along a line and then use this information for both locating curvature maxima, in order to extract the elementary parts forming the shape, and describing the shape of each part by some encoding of its curvature [15].

Unfortunately, applying the mathematical definition of curvature, may result in detecting many curvature maxima not corresponding to perceptually relevant points, eventually providing different descriptions for similar shapes.

To solve this problem, we developed a method derived from an analogy with the primate visual system for selecting the best scale at which the electronic ink of the handwriting should be described [12]. According to this analogy, the method computes a multiscale features maps by evaluating the curvature along the ink at different levels of resolution and arranges them into a pyramidal structure. Then, feature values extracted at different scales are combined in such a way that values that locally stand out from their surrounds are enhanced, while those comparable with their neighbours are suppressed. A saliency map is eventually obtained by combining those features value across all possible scales. Such a map is then used to select a representation that is largely invariant with respect to non significant shape variations encountered in handwriting.

Once the appropriate scale has been selected, the arclength representation [13] of the electronic ink at that scale is considered. This representation is a function $\alpha(\lambda)$ where $\lambda$ is the curvilinear abscissa of a point, and $\alpha$ is the angle of the tangent to the curve at that point with respect to the horizontal axis. The segmentation of the word into elementary strokes is performed by locating the curvature maxima on the arclength representation at the selected scale. The final description of the handwriting shape is given in terms of a set of strings each encoding the curvature changes relative 
to each elementary stroke. To this aim, the actual values of $\alpha(\lambda)$ are quantized into 16 intervals and each interval encoded by one of the letter of the subset [A-P] in such a way that the letter A corresponds to the first interval (from 0 to $2 \pi / 16$ ), the letter B to the second one (from $2 \pi / 16$ to $2 * 2 \pi / 16$ ) and so on, counter clockwise. By this encoding, the shape of the word is described by a string of characters that represents the desired set of features.

Figure 1 reports the electronic ink of a word, the segmentation points that allow to split the original word into elementary strokes and the strings encoding each elementary strokes.

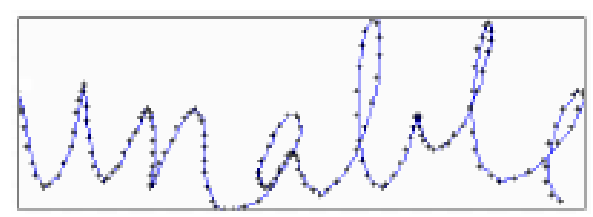

a)

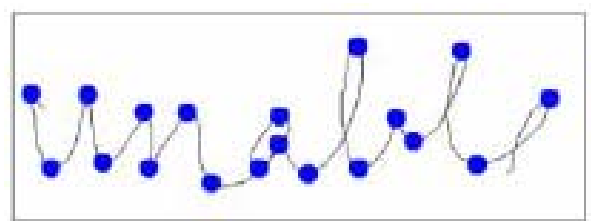

b)

J-MMNO-CDDEC-MMNO-CDD-NMM-ADDC-NMMNP-BBCDE-JKLN-CA-NPCCDDDEE-HLMMMMNO-CDD-OO-BCDDDE-LLMMMNN- ABCCDF-KLLL

c)

Fig. 1. a) The original input. b) The segmentation of the ink in elementary strokes. c) The sequence of strings encoding the change of curvature along the curve at the selected resolution: hyphens correspond to segmentation points.

\section{Weighted Edit Distance (WED)}

The basic idea the Weighted Edit Distance is based upon is that of avoiding the introduction or the deletion of symbols in the strings to compare by stretching the shortest string in such a way that each of its symbol is compared with one or more symbols of the other, depending on the ratio $r$ between the lengths of the two strings.

To formally describe the algorithm for computing WED, let us denote with S1 and S2 the two strings to compare, with S1[i] (S2[j]) the i-th (j-th) symbol in the string $\mathrm{S} 1$ (S2), and with Ls1 and Ls2 the lengths of S1 and S2, respectively. Let us also assume that $\quad \mathrm{Ls} 1 \geq \mathrm{Ls} 2$. Finally, let us denote with $\mathrm{D}(\mathrm{x}, \mathrm{y})$ the function that assigns a cost to the substitution of symbol $\mathrm{x}$ with symbol $\mathrm{y}$. In this study $\mathrm{D}(\mathrm{x}, \mathrm{y})$ is equal to the alphabetical distance between $\mathrm{x}$ and $\mathrm{y}$, i.e. $\mathrm{D}(\mathrm{A}, \mathrm{B})=1, \mathrm{D}(\mathrm{A}, \mathrm{C})=2$, and so on. 
The algorithm for computing WED is the following:

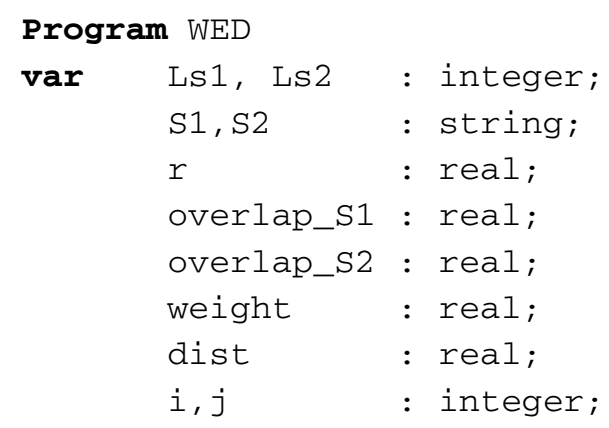

begin

get strings $\mathrm{s} 1$ and $\mathrm{s} 2$ and their lengths dist : $=0$;

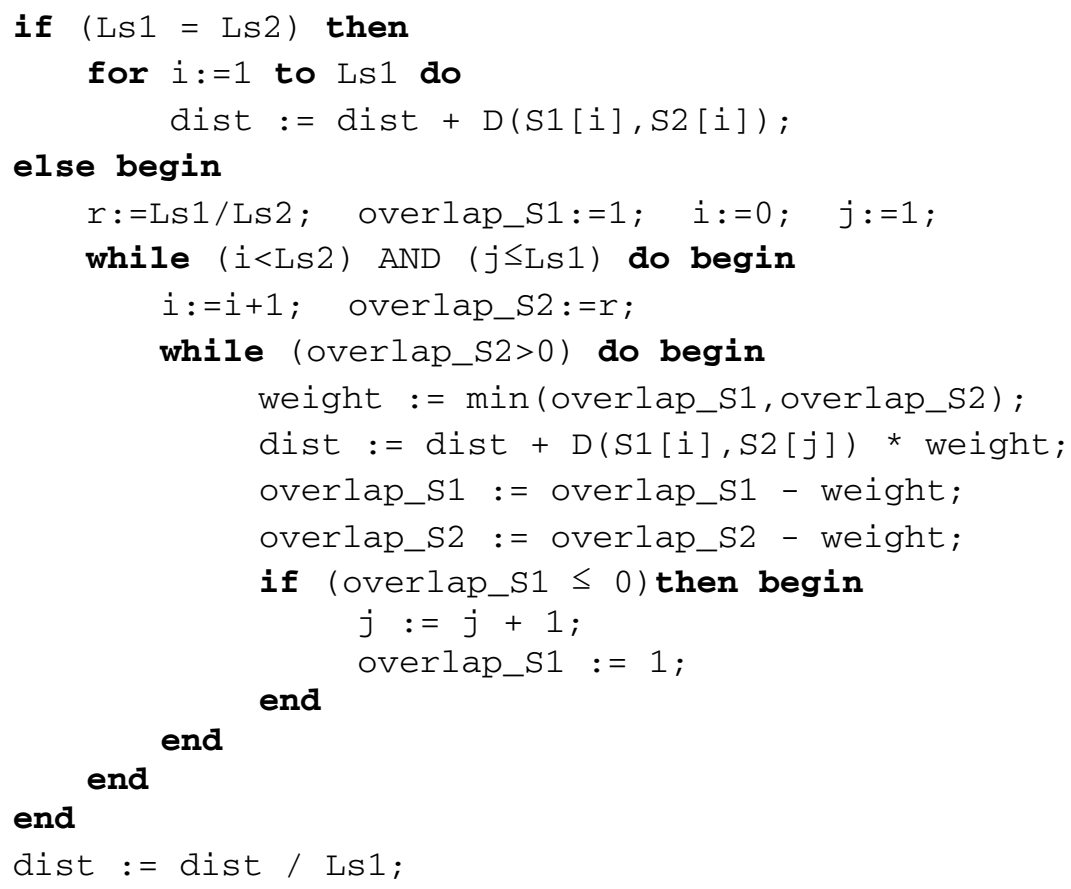

The variable overlap_S1 dynamically indicates the percentage of the current symbol in S1 which has not been exploited so far in the comparison with the symbols in $\mathrm{S} 2$. The values assumed by this variable, obviously range from 1 to zero.

The variable overlap_S2 dynamically indicates if the current symbol in S2 has been completely exploited in the comparison with the symbols in S1. The values assumed by this variable, range from $r$ to zero. When the variable overlap_S2 assumes a value greater than or equal to one, it indicates that there are more symbols in 
S1 that must be compared with the current symbol in S2: as a consequence, the value of overlap_S1 is assigned to the variable weight, the current symbol in S1 is compared with the current symbol in S2 and then the next symbol in S1 is selected. When the variable overlap_S2 assumes a value less than one, such a value represents the residual percentage of the current symbol in $\mathrm{S} 2$ that has not been exploited. It also indicates that the current symbol in S1 is the last one that must be compared with the current symbol in S2. In this situation the value of overlap_S2 is assigned to the variable weight, the current symbol in S1 is compared with the current symbol in S2 and then the next symbol in $\mathrm{S} 2$ is selected.

Summarizing, the edit distance is computed by adding at each step the cost of substitution of the current pair of symbols multiplied by the current value of the weight: this process is iterated until both symbols in S1, and symbols in S2 have been completely exploited. Finally the value of the distance is normalized by Ls1.

\section{Experiments and Discussion}

In order to validate the proposed edit distance technique, a set of experiments have been performed for comparing our results with those obtained by using the Normalized Edit Distance and the Markov Edit Distance. A further experiment has been eventually performed to show some preliminary results obtained in the framework of our on-line handwriting recognition system.

The aim of the first set of experiments is that of evaluating how WED is continuous with respect to the perception, i.e. how the distance variations measured by WED are consistent with the perceived entity of the modifications as the shape of an object is changed smoothly. Figure 2 illustrates the shape we have used for this experiments, while figure 3a)-c) plots the distance between one of the shape shown in figure 2 (shape 1, 4 and 7, respectively) and all the other ones by using WED, NED and MED. For the sake of comparison, all the shapes are composed by the same number of points and consequently are described by strings having the same length. The plots show that WED always exhibits a very consistent behavior with the variation of shape. In particular, its linear trend reflects the perceived continuous variations among the considered shapes. In contrast, NED is linear in the plot of figure 3a), but not in that of figure $3 \mathrm{c}$ ), that has been obtained by considering the distances among the shape in the reverse order with respect to that of figure $3 a$ ). MED exhibits only a

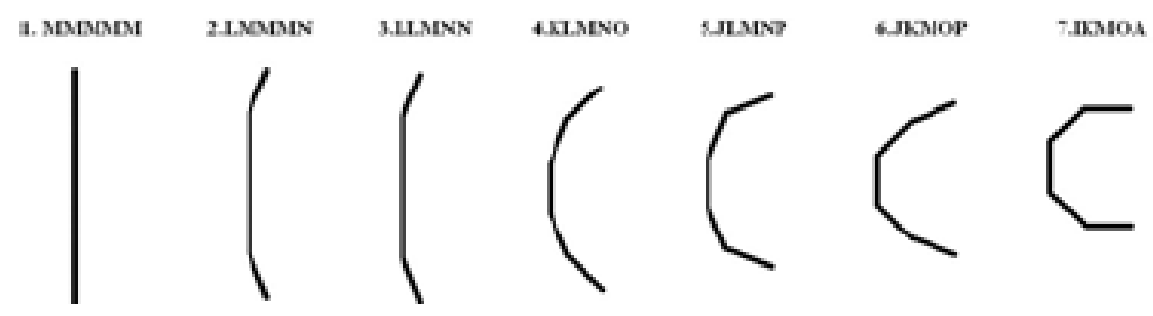

Fig. 2. The "continuum" of shapes used in the first experiment 
partial linearity, in that for some shapes there are no difference in the measured distance. Eventually, NED is non symmetric when the same amount of changes occurs on both side of the reference shape.

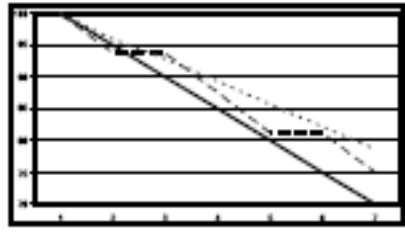

a)

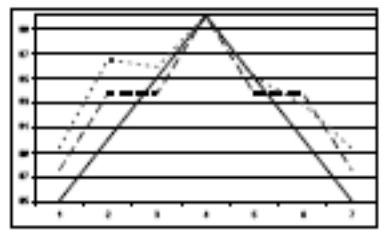

b)

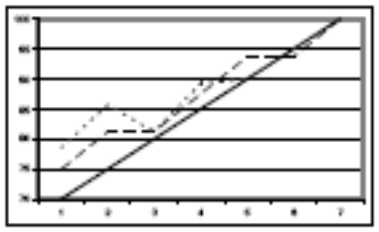

c)

Fig. 3. The distances among the shapes of figure 2 with repect to: a) shape 1 ; b) shapes 4 ; c) shape 7. In the plots, continuous lines represent WED, dashed lines MED, dotted lines NED. a)

In the second experiment, we have considered 100 words from a lexicon containing pairs of words sharing at least one bigram or trigram. The words, extracted from a database of cursive words produced in our lab, were processed and the distances among the obtained strings measured according to WED. The strings belonging to different words and corresponding to the minimum distances were eventually concatenated to obtain the best common sequence of strings. Figure 4a) shows the ink corresponding to such a sequence for two fragments of the words "ceramica" (ceramic) and "cerato" (waxed), while figure 4b) plots the distances among the corresponding strokes of the two words. As the figure illustrates, the best common sequence of strings corresponds to the most similar fragments of ink.
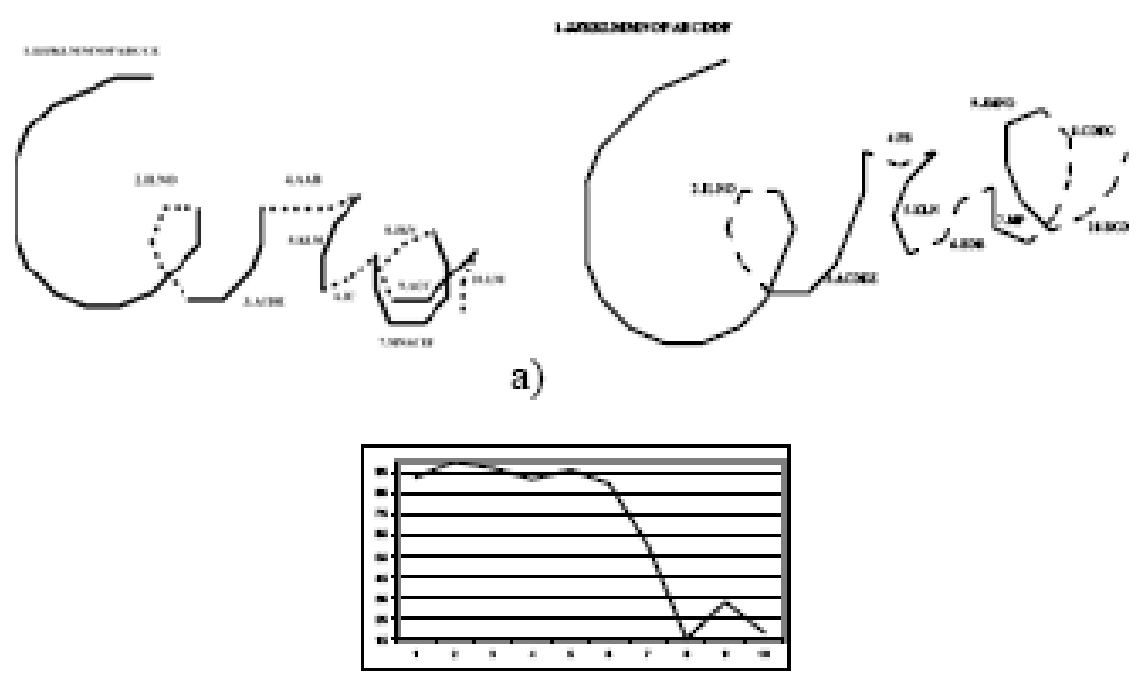

b)

Fig. 4. The application of WED to handwriting shape. a) Ink strokes and the corresponding strings; b) high values correspond to similar shapes (strokes 1 to 6 ) 
The experiments reported here suggest that the proposed distance is a promising tool toward a quantitative measure of the elusive concept of shape similarity. Further experiments on a larger database, together with a formal assessment of the metric properties of WED, are however needed in order to confirm the behavior observed during the experiments performed in this study.

\section{References}

1. Durbin, K., Eddy, S., Krogh, A., Mitchison, G.: Biological Sequence Analysis: Probabilistic Models of Proteins and Nucleic Acids. Cambridge Univ. Press (1997).

2. Cover, T.M., Thomas, J.A.: Elements of Information Theory, second ed. Wiley (1991).

3. Huang, X., Acero, A., Hon, H.: Spoken Language Processing. Prentice Hall (2001).

4. Tsay, Y.T., Tsai, W.H.: Attributed String Matching Split-and- Merge for On-Line Chinese Character Recognition, IEEE Trans. on Pattern Analysis and Machine Intelligence, vol. 15, no. 2 (1993), 180-185.

5. Ratha, N., Chen, S., Karu, K., Jain, A.K.: A Real-Time Matching System for Large Fingerprint Databases, IEEE Trans. on Pattern Analysis and Machine Intelligence, vol. 18, no. 8 (1996) 799-813.

6. Levenstein, A.: Binary Codes Capable of Correcting Deletions, Insertions and Reversals, Soviet Physics-Doklandy, vol. 10 (1966).

7. Okuda, T., Tanaka, E., Kasai, T.: A Method of Correction of Garbled Words Based on the Levenstein Metric, IEEE Trans. on Computers, (1976), 172-177.

8. Wagner, R.A., Fisher, M.J.: The String to String Correction Problem, Journal of ACM, vol. 21, no. 1 (1974), p. 168-173.

9. Marzal, A., Vidal, E.: Computation of Normalized Edit Distance and Applications, IEEE Trans. on Pattern Analysis and Machine Intelligence, vol. 15, no. 9 (1993), 926-932.

10. 10. Wei, Jie: Markov Edit Distance, IEEE Trans. on Pattern Analysis And Machine Intelligence, vol. 26, no. 3 (2004), 311-321.

11. De Stefano, C., Guadagno, G., Marcelli A.: A Saliency-Based Segmentation Method for On-Line Cursive Handwriting, International Journal of Pattern Recognition and Artificial Intelligence, vol.18, no. 6 (2004), 1139-1156.

12. De Stefano, C., Garruto, M., Marcelli, A.: A saliency-based multiscale method for on-line cursive handwriting shape description", Proc. of the 9th International Workshop on Frontiers in Handwriting Recognition 2004 (IWFHR-9), Tokyo, Japan, October, 26-29, 2004, 124-129.

13. Pavlidis, T.: Structural Pattern Recognition, Springer-Verlag, 1977.

14. Fischler, M.A., Bolles, R.C.: Perceptual organization and curve partitioning, IEEE Trans. on Pattern Analysis and Machine Intelligence, vol. 8, no. 1 (1986), 100-105.

15. Marshall, S.: Review of shape coding techniques, Image and Vision Computing, vol. 7, no. 4 (1989), 281-294. 